\title{
Atomic-level viscosity distribution in the hydration layer
}

$\operatorname{AUTHOR}(S)$ :

Umeda, Kenichi; Kobayashi, Kei; Minato, Taketoshi; Yamada, Hirofumi

\section{CITATION:}

Umeda, Kenichi ... [et al]. Atomic-level viscosity distribution in the hydration layer. Physical Review Letters 2019, 122(11): 116001.

\section{ISSUE DATE:}

2019-03-22

URL:

http://hdl.handle.net/2433/241390

\section{RIGHT:}

(C) 2019 The American Physiological Society; This is the accepted version of the article, which has been published in final form at http://doi.org/10.1103/PhysRevLett.122.116001. 


\title{
Atomic-Level Viscosity Distribution in the Hydration Layer
}

\author{
Kenichi Umeda, ${ }^{1,2,3}$ Kei Kobayashi, ${ }^{1}$ Taketoshi Minato, ${ }^{4}$ and Hirofumi Yamada ${ }^{1, *}$ \\ ${ }^{1}$ Department of Electronic Science and Engineering, Kyoto University, Katsura, Nishikyo, Kyoto 615-8510, Japan \\ ${ }^{2}$ Department of Advanced Material Science, The University of Tokyo, Kashiwa, Chiba, 277-8561, Japan \\ ${ }^{3}$ Nano Life Science Institute, Institute for Frontier Science Initiative, Kanazawa University, \\ Kakuma, Kanazawa, Ishikawa, 920-1192, Japan \\ ${ }^{4}$ Office of Society-Academia Collaboration for Innovation, Kyoto University, Katsura, Nishikyo, Kyoto 615-8510, Japan
}

(Received 8 January 2019; published 21 March 2019)

\begin{abstract}
The viscosity of solvation structures is crucial for the development of energy-efficient biofunctional and electrochemical devices. Elucidating their subnanoscale distributions can cause the formation of a sustainable energy society. Here, we visualize the site-specific three-dimensional damping distribution on a $\mathrm{CaCO}_{3}$ surface composed of binary ion species using ultra-low-noise frequency modulation atomic force microscopy. With the support from molecular dynamics simulation, we found a strikingly large damping at the calcium sites, which demonstrates the capability of this methodology to visualize atomicscale viscosity in the hydration layers. Our finding will expedite the evolutions of various functional devices.
\end{abstract}

DOI: 10.1103/PhysRevLett.122.116001

The peculiar nature of atomic-level hydration water dynamics on surfaces has enabled the development of various energy-efficient devices [1]. Particularly, the viscosity and diffusion of water in the hydration structures assume important roles in biofunctions [2,3] and electrochemical reactions [4] because they dominate the stability of biomolecules and the transport velocity of electrolytes. Therefore, characterizing their subnanoscale three-dimensional (3D) distributions is crucial for industrial applications as well as fundamental scientific interest.

Recently, we developed an ultra-low-noise liquid 3D frequency-modulation atomic force microscopy (FM-AFM) technique, which has enabled the evaluation of hydration distributions [5-8]. Furthermore, FM-AFM has an advantage in separately detecting the conservative and dissipative forces as frequency shift $(\Delta f)$ and dissipative damping, respectively [9-12]. Dissipation measurements under vacuum conditions have been prevalently used to evaluate the viscoelasticities and structural stabilities of surface species [9-12]. Meanwhile, in liquids, it has been primarily exploited to study the hydrodynamic squeeze effect [13], the fluid viscosity augmented near surfaces [14-17], and interfacial energy [18]. Despite the usefulness of the damping measurements, whether the intrinsic dissipative damping profiles in solvation measurements exhibit an oscillatory behavior has been extensively debated because of several issues encountered while performing the quantitative measurements in liquids [14-16,19]. Specifically, the surface force measurements [17] cannot detect intrinsic damping profiles due to the inevitable macroscopic confinement effect $[6,20]$. Therefore, AFM utilizing an atomically sharpened tip must be used; however, most of the experiments have been conducted using tapping-mode AFM with piezo excitation (causing spurious peaks), which cannot circumvent the entanglement between the conservative and dissipative forces [14-16].

We previously solved this problem using an atomicresolution spurious-free 3D FM-AFM instrument that can completely decouple their forces, which further revealed a monotonic damping profile [6]. Nevertheless, site-specific damping measurements on several atom-chemical species at different heights have yet to be achieved. In the present study, we demonstrate this challenging experiment using a recently developed nondestructive observation protocol $[7,8]$. Consequently, by combining molecular dynamics (MD) simulations with our AFM observations, we discover that site-specific damping with a shoulderlike peak found in AFM data reflects the locally reduced water self-diffusion coefficient $\left(D_{w}\right)$.

To reveal the relationship between the damping and surface chemical-atom species, we employed a calcite (104) surface [18,21-27], exposing a binary structure of $\mathrm{Ca}$ and $\mathrm{CO}_{3}$ sites arranged in a grid pattern as illustrated in Fig. 1(a). Among the three oxygen atoms in the $\mathrm{CO}_{3}$ ions, one protrudes from the surface, whereas one is depressed into the surface. As described later, two equivalent $\mathrm{CO}_{3}$ 's in each unit cell are frequently observed as protrusions that differ in height or shape in the AFM imaging in a vacuum as well as in water $[21,22]$. Although this reconstruction in the apparent surface is called the row-pairing structure since the two protruding oxygen rows appear as a pair, it is currently considered to be a tip-induced artifact because all the evidence has totally relied on only the AFM measurements [21,22]. 


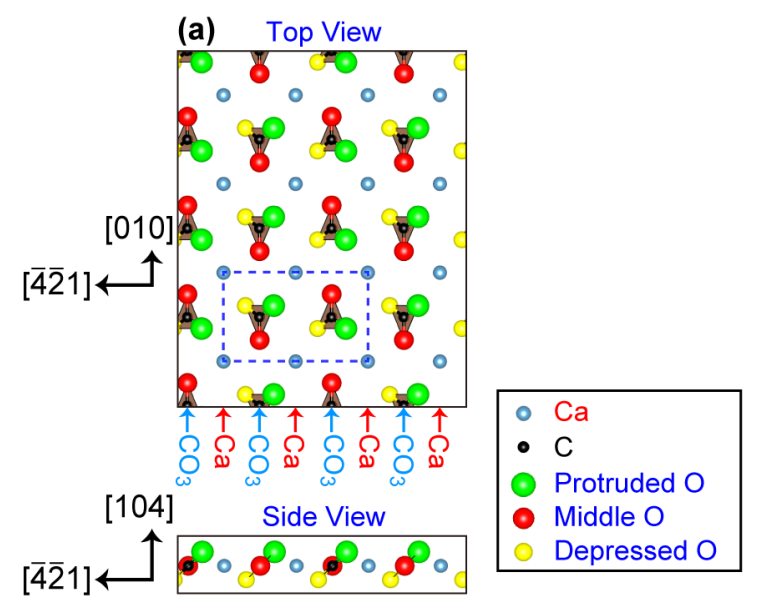

(b) 3D Force map

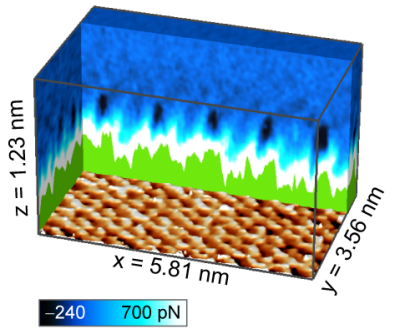

(c) 3D damping map

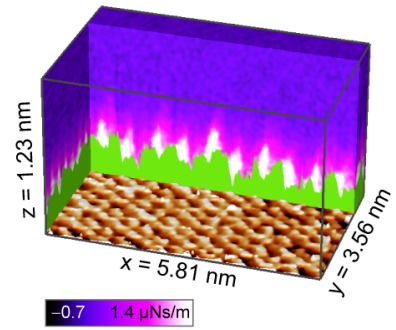

FIG. 1. Overview of the study. (a) Top and side views of the crystal structures of calcite (104). The broken blue rectangle represents the unit cell $\left(0.81 \times 0.50 \mathrm{~nm}^{2}\right)$. (b),(c) Representations of simultaneously acquired 3D conservative force (b) and damping (c) maps, in which the bottom faces show reconstructed constant $\Delta f$ images at $500 \mathrm{~Hz}$.

Since the details of the 3D experiment and simulation have been elaborated in our previous reports $[7,8]$, we now briefly outline them. We used a home-built FM-AFM system with a LabVIEW PXI controller (National Instruments). To realize the complete disentanglement of the conservative and dissipative forces, maintaining a constant oscillation amplitude while tracking the resonance frequency is required. To fulfill this criterion, we used two independent feedback loops, i.e., self-oscillation and constant-amplitude feedback circuits. Moreover, to circumvent the problem of the "forest of peaks" in liquids, we employed a spurious-free photothermal excitation setup. We used a rectangular cantilever (Nanosensors: PPP$\mathrm{NCHAuD}$ ), whose spring constant and resonance frequency in water were $30 \mathrm{~N} / \mathrm{m}$ and $140 \mathrm{kHz}$, respectively, with the oscillation amplitude of $0.5 \mathrm{~nm}_{p-p}$. A high-purity single crystal of calcite produced in Madagascar, purchased from a crystal trading company (The Stone of WAKOU), was cleaved using a cutting knife immediately prior to the experiment. The experiment was conducted in ultrapure water to exclude any effects caused by adsorbed ions. 3D $\Delta f$ and excitation voltage maps, which contain $128 \times 27 \times$ 105 pixels $\left(5.81 \times 3.56 \times 1.23 \mathrm{~nm}^{3}\right)$ in $X Y Z$, were simultaneously acquired for $1 \mathrm{~min}$. We analyzed both the experimental and simulated 3D data using a home-built program developed in Visual Basic .NET (Microsoft).

To detect the minute damping signal, we acquired the 3D data [Figs. 1(b),1(c)], also see the movie in the Supplemental Material [28]) with a threshold value of $5 \mathrm{kHz}$, which is remarkably higher than the typical value of $1 \mathrm{kHz}[6,8]$. The apparent surface rendered in the crosssectional maps (boundaries between the white and green regions) of Fig. 1(b) was somewhat rumpled because of several vertical positions of the atoms, which indicated that our established nondestructive observation protocol $[7,8]$ is essential for the tip to obtain deep access to the individual sites. To compare the damping acquired on the individual sites at the same tip-sample distance, we first reconstructed a constant $\Delta f$ plane as a topographic image that could have been obtained slightly above the threshold plane. The topography presented in Fig. 2(a) depicts a clear atomic resolution with characteristic row-pairing bright spots, forming a stripelike shape along the [010] direction. In line with previous studies [21-23], we assumed the bright spots as the protruded oxygen (pO) sites as seen in the overlaid crystal model. Figure 2(b) depicts a damping map reconstructed in the same constant $\Delta f$ plane in Fig. 2(a). In contrast to the topography, this did not show the row-pairing structure. By overlaying the crystal model, we identified the bright spots in the damping image as the Ca sites.

To analyze the experimental data, we performed MD simulations. We retrieved a calcite crystal structure from the American Mineralogist Crystal Structure Database and
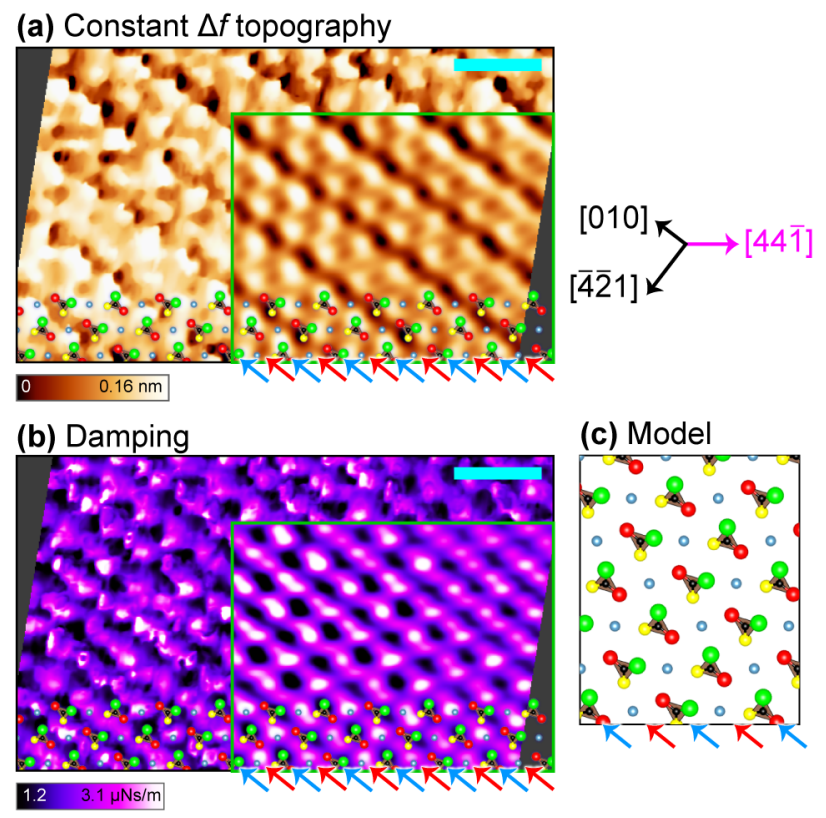

FIG. 2. Constant- $\Delta f$ atomic resolutions. (a)-(c) Experimental reconstructed topography (b) and damping image (b) at the contour surface at $\Delta f=4 \mathrm{kHz}$, which roughly corresponds to $0.6 \mathrm{nN}$, and crystal structure (c). Scale bars, $1 \mathrm{~nm}$ (a)-(c). 
constructed a $(2 \times 4)$ supercell exposing the (104) plane $\left(1.62 \times 2.00 \mathrm{~nm}^{2}\right)$ with overlaying $800-1000$ water molecules (Fig. S1 in the Supplemental Material [28]). A dynamics simulation was performed with a force field optimized for calcite [24] in the $N V T$ ensemble using Forcite (Dassault Systèmes BIOVIA). After equilibration, the calculation was conducted for $70 \mathrm{~ns}$ which was sufficiently long since we confirmed that increasing the simulation time greater than $1 \mathrm{~ns}$ simply improved the signal-to-noise ratio of the data. Note that the simulation time was still 2 orders of magnitude shorter than the timescale of the tip oscillation cycle. Since we did not perform the MD simulations to directly simulate the dynamic (nonequilibrium) effects caused by the tip movement, e.g., adhesion hysteresis [29], the difference in the timescale was not problematic.

We first obtained a time-averaged 3D water density map, and assigned numbers to the density peaks in the averaged profile. We then calculated 3D force data using a solvent tip approximation model [30], which has accurately reproduced the experimental force data $[7,8]$. In the simulated average force profile [Fig. S2(b) in the Supplemental Material [28]], the second and third force peaks are slightly split, while the experimental ones were observed as a shoulder (Fig. S3 in the Supplemental Material [28]). Therefore, we call these layers the second (third) layer in the following discussion.

We compared the reconstructed constant-height force maps in the individual layers. That in the second (third) layer [Fig. 3(a)] showed the rows of the bright spots corresponding to the $\mathrm{pO}$ sites. Note that the rowpairing structure was not observed at this height and the contrast features resemble those in the simulated force image [Fig. 3(b)]. This result is reasonable because the tipsample interaction should increase at the $\mathrm{pO}$ sites because of the reduced tip-sample distance. Meanwhile, the corresponding damping map [Fig. 3(c)] showed bright spots at the $\mathrm{Ca}$ sites, whose patterns resemble the inverse contrast of the constant-height force map [Fig. 3(a)] but showed the row-pairing structure.

Next, we also reconstructed the constant-height maps in the fourth layer in Figs. 3(e),3(f). Both the experimental and simulated images exhibited a similar contrast feature, e.g., their dark spots were observed on the pO sites. In the experimental result, we found that these dark spots were observed in different darkness at every other row, forming the row-pairing characteristics. The corresponding damping image in Fig. 3(g) also showed the row-pairing structure. Although this image was ambiguous compared to that of the second (third) layer [Fig. 3(c)], the filtered image indicated that the distribution of bright spots is similar to that in the second layer with a minor deviation.

From the early days of FM-AFM, the origin of the damping has been frequently debated [9-12] and is currently regarded as a hysteresis caused by the rearrangement of tip-sample atoms occurring synchronously with the tip oscillation cycle. As for calcite in a vacuum, the $\mathrm{CO}_{3}$ site shows a greater damping than the $\mathrm{Ca}$ site due to

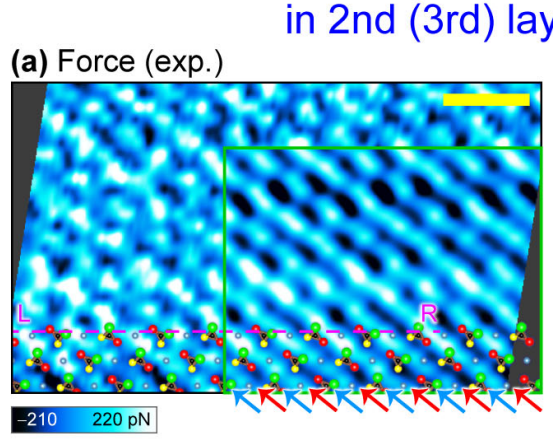

(c) Damping (exp.)

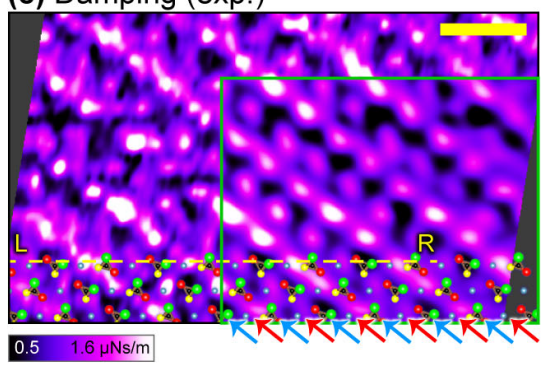

(b) Force (sim.)

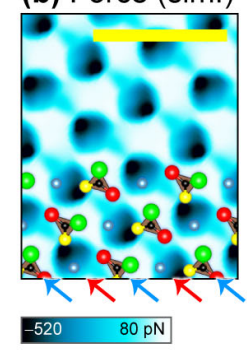

(d) $D_{w}$ (sim.)

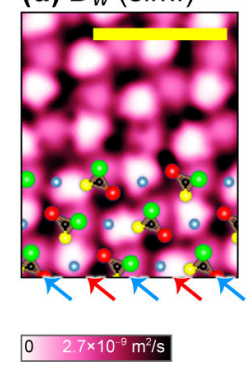

in 4th layer
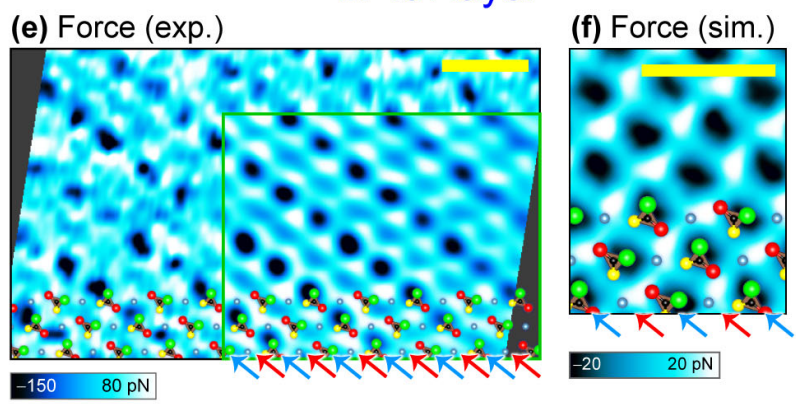

(g) Damping (exp.)
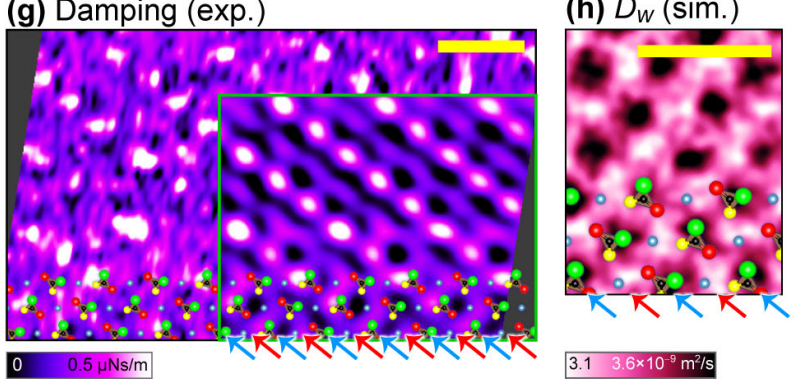

FIG. 3. Constant-height atomic resolutions. (a)-(h) Horizontal distributions of experimental (a),(c),(e),(g) and simulated (b),(d),(f),(h) forces and (a),(b),(e),(f) damping (c),(g), and $D_{w}(\mathrm{~d}),(\mathrm{h})$ in the second (third) (a)-(d) and fourth (e)-(h) layers. The insets in (a),(c),(e),(g) and show Fourier-filtered images. The inverted color-scale bar is used in (d),(h). Scale bars, $1 \mathrm{~nm}(\mathrm{a})-(\mathrm{h})$. 
the rotation of the $\mathrm{CO}_{3}$ ion induced by the direct tipsample interaction $[25,26]$. However, our results in water exhibited the damping contrast even relatively far from the surface and the higher damping signal at the Ca site than at the $\mathrm{CO}_{3}$ site. Moreover, the dissipated energy was 2000-3000 meV/cycle, about an order of magnitude greater than $180 \mathrm{meV} /$ cycle in the vacuum [25]. These facts compelled us to consider the indirect interaction via water molecules [31].

Since the water molecules diffuse much faster than the tip oscillation velocity, the hydration structures manifest in the conservative force data. Therefore, the local variation in the damping has not been discussed in the previous experimental studies [5-8]. However, a recent theoretical study showed that $D_{w}$ in the space atomically confined between a cluster tip and a sample surface is reduced by up to 3 orders of magnitude [31]. Furthermore, other theoretical research revealed that the macroscopic confinement effect further reduces $D_{w}$ by 3 orders of magnitude [29]. These nanolevel and macroscopic confinements could cooperatively reduce $D_{w}$ to be the same order as the tip oscillation velocity, which possibly produce the damping signal with the atomic-scale variation.

Therefore, to elucidate the increase in damping at the $\mathrm{Ca}$ sites, we calculated $D_{w}$ using the Einstein relation $[3,32,33]$. Since this formulation cannot be simply applied to the interfacial water, we divided the simulation volume to $0.033-\mathrm{nm}$ cubic units and averaged the mean square displacement of water oxygen atoms in each unit in the linear diffusion regime (1 ps), following a previous study
[33], where a good agreement with experimental results was demonstrated. We examined the horizontal $D_{w}$ map in the second (third) and fourth layers in Figs. 3(d) and 3(h), respectively, which exhibited the bright spots at the $\mathrm{Ca}$ sites, as similarly observed in the damping maps. This indicates that the water molecules were restricted in the hollow structures surrounded by the $\mathrm{pO}$ sites, which causes the strong damping due to the reduced $D_{w}$. This is consistent with our recent study that showed the reduction of $D_{w}$ by the steric restrictions caused by the surfaces [8]. In addition, since the topographic images depicted the rowpairing structure, the tip apex seems to be terminated by the anisotropic $\mathrm{CO}_{3}$ ion instead of the isotropic $\mathrm{Ca}$ ion. In this case, the dipole moment of the water molecule on the $\mathrm{CO}_{3}$ tip (negatively charged) directs upward while that on the $\mathrm{Ca}$ atom (positively charged) on the sample directs in the same direction. Therefore, the water molecule is strongly trapped between the tip and sample, which further reduces the water relaxation time.

To gain deeper insight into the damping phenomena, we subsequently examined the vertical maps extracted from the 3D data along the broken lines in Figs. 3(a),3(c), which are roughly across the $\mathrm{Ca}$ and $\mathrm{pO}$ sites. Both the experimental and simulated force maps in Figs. 4(a),4(b) depict periodic attractive dark spots above the second (third) layers between the $\mathrm{Ca}$ and $\mathrm{pO}$ sites. However, the experimental map exhibited those only at every other row, and two types of Ca sites with different apparent heights, possibly because the finite radius and anisotropy of the tip were not incorporated in the simulation. Note that the dark spots (a) Force (exp.)

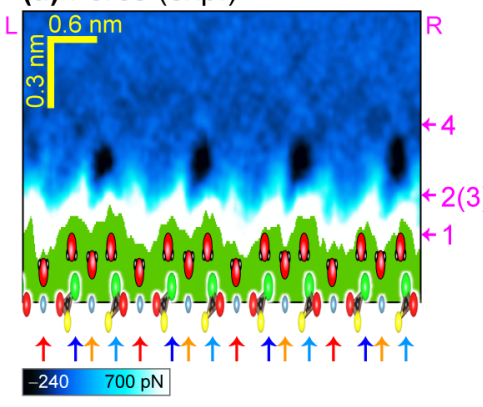

(e) Force profile (exp.)

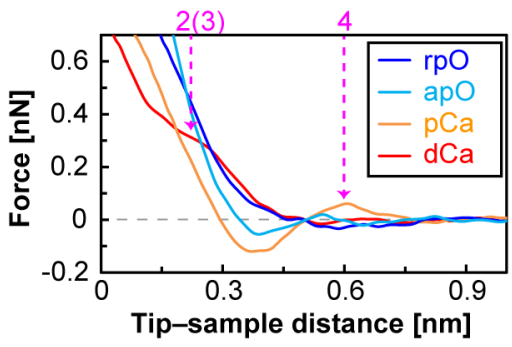

(b) Force (sim.)

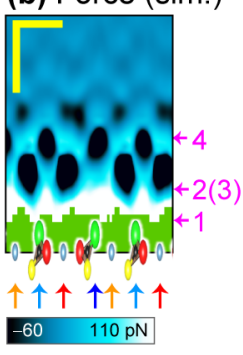

(c) Damping (exp.)

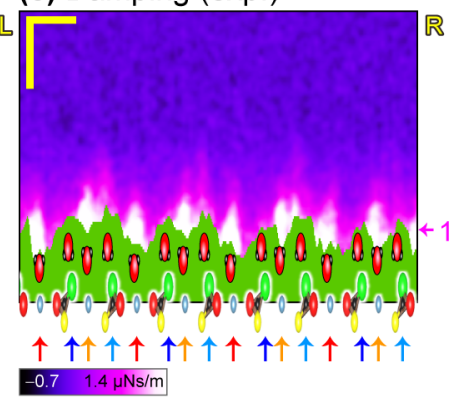

(d) $D_{W}(\operatorname{sim}$.

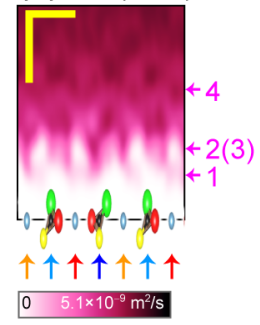

(f) Damping profile (exp.)

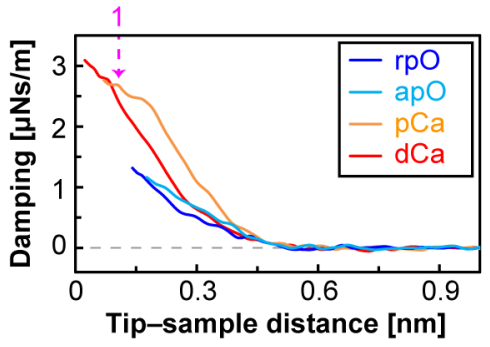

FIG. 4. Site dependencies of force and damping. (a)-(d) Experimental (a),(c) and simulated (b),(d) vertical force (a),(b), damping (c), and $D_{w}(\mathrm{~d})$ maps on a calcite surface extracted from the 3D data along the broken lines in Figs. 3(a) and 3(c), respectively. The inverted color contrast is used in (d). (e),(f) Experimental force (e) and damping (f) profiles at the individual sites of calcite. Scale bars in (a)-(d) are the same. 
in the fourth layer above the $\mathrm{pO}$ sites predicted in the simulation were not experimentally observed.

Meanwhile, the damping map in Fig. 4(c) rendered a completely different contrast feature from the conservative force map, i.e., a spikelike local increase at the $\mathrm{Ca}$ sites, which clearly indicated that the damping maps provide information distinguishable from the conservative force maps. Moreover, the vertical $D_{w}$ map in Fig. 4(d) showed a similar contrast feature, a remarkable local decrease at the $\mathrm{Ca}$ sites.

For a more quantitative analysis, we examined the profiles on the individual sites [Figs. 4(e),4(f)]. Based on the experimentally observed characteristics, we refer the four sites as the darker repulsive $\mathrm{pO}(\mathrm{rpO})$, less dark attractive $\mathrm{pO}(\mathrm{apO})$, the apparently protruded $\mathrm{Ca}(\mathrm{pCa})$, and depressed $\mathrm{Ca}(\mathrm{dCa})$ sites. The $\mathrm{rpO}$ and $\mathrm{dCa}$ conservative force profiles showed a monotonic increase caused by the interaction with the first hydration layer, but a minute shoulderlike second hydration layer was observed only in the $\mathrm{dCa}$ profile. The $\mathrm{pCa}$ profile showed the fourth hydration peak. The apO profile also showed a slight oscillatory behavior because, as clearly deduced from Fig. 4(a), the anisotropic tip simultaneously interacts with the hydration structures on the $\mathrm{pCa}$ sites.

In the damping profiles, only the $\mathrm{pO}$ profiles showed a simple monotonic increase whereas the Ca profiles showed a shoulderlike peak with a 2 times larger signal. We also reconstructed a correlation graph between $\Delta f$ and damping, which proves that the local damping was not affected by $\Delta f$ (Fig. S4 in the Supplemental Material [28]). In a previous study [6], the damping profile obtained on mica showed a monotonic behavior; however, we found that after approaching the tip closer to the mica surface, the profiles exhibit the existence of a shoulderlike peak (Fig. S5 in the Supplemental Material [28]). The peak in the damping profile possibly corresponds to the minima in the $D_{w}$ profile [Fig. S2(c) in the Supplemental Material [28]]. Since $D_{w}$ is closely related to the viscosity, which is essential in biological and electrochemical processes [2-4], the atomic-level $D_{w}$ measurement is crucial for progress in such fields.

To summarize, we visualized an atomic-scale hydration structure and the accompanying damping or viscosity distribution on a calcite (104) surface via liquid 3D FMAFM. The damping maps showed stronger signals at the $\mathrm{Ca}$ sites than at the $\mathrm{pO}$ sites. MD simulation revealed that this contrast reflects the locally reduced $D_{w}$ near the surface. Furthermore, we revealed that the damping profiles show the presence of a shoulderlike feature when the tip strongly interacted with the first hydration layer. The atomic-level damping measurement provides knowledge about the local viscosity distribution, which is complementary to the conservative hydration measurement. This study will be a milestone for elucidating the relationship between the water viscosity and biofunctions and electrochemical reactions.
This study was supported by KAKENHI, Japan Society for the Promotion of Science (Grants No. 24221008, No. 25286057, No. 15K17467, and No. 16J01165); Foundation Advanced Technology Institute; the Murata Science Foundation; and Nanotech Career-up Alliance. The computer resources for the MD simulation were provided by the SuperComputer System, Institute for Chemical Research, Kyoto University. K. U. would like to thank Professor Masataka Nagaoka at Nagoya University for advice on the simulations.

*Corresponding author. h-yamada@kuee.kyoto-u.ac.jp

[1] V. R. Stamenkovic, D. Strmcnik, P. P. Lopes, and N. M. Markovic, Energy and fuels from electrochemical interfaces, Nat. Mater. 16, 57 (2017).

[2] P. Ball, Water as an active constituent in cell biology, Chem. Rev. 108, 74 (2008).

[3] S. Pronk, E. Lindahl, and P. M. Kasson, Dynamic heterogeneity controls diffusion and viscosity near biological interfaces, Nat. Commun. 5, 3034 (2014).

[4] S. Y. Wei, Z. Cheng, P. Nath, M. D. Tikekar, G. J. Li, and L. A. Archer, Stabilizing electrochemical interfaces in viscoelastic liquid electrolytes, Sci. Adv. 4, eaao6243 (2018).

[5] T. Fukuma, Y. Ueda, S. Yoshioka, and H. Asakawa, AtomicScale Distribution of Water Molecules at the Mica-Water Interface Visualized by Three-Dimensional Scanning Force Microscopy, Phys. Rev. Lett. 104, 016101 (2010).

[6] A. Labuda, K. Kobayashi, K. Suzuki, H. Yamada, and P. Grütter, Monotonic Damping in Nanoscopic Hydration Experiments, Phys. Rev. Lett. 110, 066102 (2013).

[7] K. Umeda, L. Zivanovic, K. Kobayashi, J. Ritala, H. Kominami, P. Spijker, A. S. Foster, and H. Yamada, Atomic-resolution three dimensional hydration structures on a heterogeneously charged surface, Nat. Commun. 8, 2111 (2017).

[8] K. Umeda, K. Kobayashi, T. Minato, and H. Yamada, Atomic-scale 3D local hydration structures influenced by water-restricting dimensions, Langmuir 34, 9114 (2018).

[9] N. Sasaki and M. Tsukada, Effect of microscopic nonconservative process on noncontact atomic force microscopy, Jpn. J. Appl. Phys., Part 2 39, L1334 (2000).

[10] L. N. Kantorovich and T. Trevethan, General Theory of Microscopic Dynamical Response in Surface Probe Microscopy: From Imaging to Dissipation, Phys. Rev. Lett. 93, 236102 (2004).

[11] N. Oyabu, P. Pou, Y. Sugimoto, P. Jelinek, M. Abe, S. Morita, R. Perez, and O. Custance, Single Atomic Contact Adhesion and Dissipation in Dynamic Force Microscopy, Phys. Rev. Lett. 96, 106101 (2006).

[12] S. A. Ghasemi, S. Goedecker, A. Baratoff, T. Lenosky, E. Meyer, and H. J. Hug, Ubiquitous Mechanisms of Energy Dissipation in Noncontact Atomic Force Microscopy, Phys. Rev. Lett. 100, 236106 (2008).

[13] I. Nnebe and J. W. Schneider, Characterization of distancedependent damping in tapping-mode atomic force microscopy force measurements in liquid, Langmuir 20, 3195 (2004). 
[14] A. Maali, T. Cohen-Bouhacina, G. Couturier, and J. P. Aime, Oscillatory Dissipation of a Simple Confined Liquid, Phys. Rev. Lett. 96, 086105 (2006).

[15] S. J. O'Shea, Comment on "Oscillatory dissipation of a simple confined liquid," Phys. Rev. Lett. 97, 179601 (2006).

[16] G. B. Kaggwa, J. I. Kilpatrick, J. E. Sader, and S. P. Jarvis, Artifact-free dynamic atomic force microscopy reveals monotonic dissipation for a simple confined liquid, Appl. Phys. Lett. 93, 011909 (2008).

[17] L. Bureau, Nonlinear Rheology of a Nanoconfined Simple Fluid, Phys. Rev. Lett. 104, 218302 (2010).

[18] K. Voïtchovsky, J. J. Kuna, S. A. Contera, E. Tosatti, and F. Stellacci, Direct mapping of the solid-liquid adhesion energy with subnanometre resolution, Nat. Nanotechnol. 5, 401 (2010).

[19] S. de Beer, W. K. den Otter, D. van den Ende, W. J. Briels, and F. Mugele, Non-monotonic variation of viscous dissipation in confined liquid films: A reconciliation, Europhys. Lett. 97, 46001 (2012).

[20] J. I. Kilpatrick, S. H. Loh, and S. P. Jarvis, Directly probing the effects of ions on hydration forces at interfaces, J. Am. Chem. Soc. 135, 2628 (2013).

[21] S. Rode, N. Oyabu, K. Kobayashi, H. Yamada, and A. Kühnle, True atomic-resolution imaging of (1014) calcite in aqueous solution by frequency modulation atomic force microscopy, Langmuir 25, 2850 (2009).

[22] P. Rahe, J. Schütte, and A. Kühnle, NC-AFM contrast formation on the calcite (1014) surface, J. Phys. Condens. Matter 24, 084006 (2012).

[23] T. Fukuma, B. Reischl, N. Kobayashi, P. Spijker, F. F. Canova, K. Miyazawa, and A. S. Foster, Mechanism of atomic force microscopy imaging of three-dimensional hydration structures at a solid-liquid interface, Phys. Rev. B 92, 155412 (2015).

[24] P. Raiteri and J. D. Gale, Water Is the Key to Nonclassical Nucleation of Amorphous Calcium Carbonate, J. Am. Chem. Soc. 132, 17623 (2010).
[25] S. Kuhn, M. Kittelmann, Y. Sugimoto, M. Abe, A. Kühnle, and P. Rahe, Identifying the absolute orientation of a lowsymmetry surface in real space, Phys. Rev. B 90, 195405 (2014).

[26] A. S. Foster, A. Y. Gal, Y. J. Lee, A. L. Shluger, and R. M. Nieminen, Dependence of the tip-surface interaction on the surface electronic structure, Appl. Surf. Sci. 210, 146 (2003).

[27] D. S. Wastl, M. Judmann, A. J. Weymouth, and F. J. Giessibl, Atomic resolution of calcium and oxygen sub lattices of calcite in ambient conditions by atomic force microscopy using qplus sensors with sapphire tips, ACS Nano 9, 3858 (2015).

[28] See Supplemental Material at http://link.aps.org/ supplemental/10.1103/PhysRevLett.122.116001 for additional experimental and theoretical data, i.e., snapshots from the MD simulation, averaged experimental and simulated profile, correlation between frequency shift and damping, 3D force map data on mica, and movie for 3D force and damping map measurement on calcite.

[29] Y. S. Leng, Hydration force between mica surfaces in aqueous $\mathrm{KCl}$ electrolyte solution, Langmuir 28, 5339 (2012).

[30] M. Watkins and B. Reischl, A simple approximation for forces exerted on an AFM tip in liquid, J. Chem. Phys. 138, 154703 (2013).

[31] M. Watkins, M. L. Berkowitz, and A. L. Shluger, Role of water in atomic resolution AFM in solutions, Phys. Chem. Chem. Phys. 13, 12584 (2011).

[32] P. Liu, E. Harder, and B. J. Berne, On the calculation of diffusion coefficients in confined fluids and interfaces with an application to the liquid-vapor interface of water, J. Phys. Chem. B 108, 6595 (2004).

[33] I. C. Bourg and C. I. Steefel, Molecular dynamics simulations of water structure and diffusion in silica nanopores, J. Phys. Chem. C 116, 11556 (2012). 